\title{
Rotwelfh).
}

Suellen and Wortjhats der Bauneripradge

unt ber verwandten Şeheimiprachen

bon

\section{Friedrich Kluge}

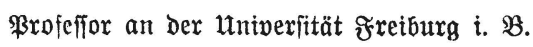

I.

Rotwelfwes Datellenbud.

Etrafburat.

Berlag von $\mathfrak{R} a r l$ J. Trübner.

1901. 
Druat von \$r. DuDiont:-ভgauberg, Straßjburg. 


\section{Seermant $\mathfrak{B a t h l}$}

in Berebrung und Danfbarfeit

gewiomet. 
